\title{
Diabetic mastopathy: A clinical and radiological challenge
}

\section{Debra Ardath Meerkotter, MB BCh, FCRad (D) SA}

Regional Imaging, St Vincents Hospital, Launceston, Tasmania, Australia

Grace Rubin, MB BCh, DA (SA), FCRad (Diag) (SA)

Department of Radiology, Helen Joseph Hospital, Johannesburg

\section{Presentation}

A 41-year-old woman presented with a lump above the right areola. She also complained of a burning and heavy sensation of the right breast of recent onset. On further history, the patient was an insulin-dependent diabetic of 35 years' duration. She had no family history of breast disease. On examination, a palpable thickening above the right areola was detected.

Bilateral mammography revealed dense fibro-glandular tissue, denser in the right retro-areolar region than the left, with right peri-areolar skin thickening. There was no discrete mass nor suspicious microcalcifications or axillary adenopathy. Ultrasound (US) demonstrated an ill-defined peri-areolar region of decreased echogenicity and posterior shadowing.

\section{Diagnosis}

Core biopsy under US guidance showed abundant thick collagen bands containing epitheloid fibroblasts and a stroma with small capillarysized and larger muscularised blood vessls, some of which contained peri-vascular lymphocytic inflammatory cells. Small ductal elements and an occasional lobule showed a peri-ductal and a peri- and intralobular lymhocytic and plasma cell infiltrate. These features were in keeping with sclerosing lymphocytic mastitis, known also as diabetic mastopathy.

\section{Discussion}

Diabetic mastopathy is an uncommon benign fibro-inflamatory breast disease. ${ }^{1}$ It may mimic cancer and as such poses a diagnostic challenge. ${ }^{2}$ Raising awareness of this condition is essential to avoid unnecessary surgical intervention and patient anxiety. ${ }^{3}$ Diabetic mastopathy accounts for less than $1 \%$ of benign breast lesions. Up to $13 \%$ of long-standing insulin-dependent diabetics are affected by diabetic mastopathy. ${ }^{4}$ The disease is not exclusive to females. ${ }^{6}$ It was first reported by Soler and Khardori in 1984. Three years later, the term 'diabetic mastopathy' was given to this entity. ${ }^{6}$

The pathogenesis is not completely understood. Three dominant mechanisms are:

- exogenous insulin may result in an inflammatory or immunologic reaction to the insulin, the vehicle or a contaminant in the vehicle ${ }^{6,7}$

- the effects of hyperglycaemia have been postulated. Hyperglycaemia results in abnormal extracellular matrix accumulation. The abnormal matrix and advanced glycosylated end products create a neoantigen that triggers a secondary auto-immune reaction and B-cell proliferation and auto-antibody formation ${ }^{6,7}$

- the role of auto-immunity must be considered. There is a link between diabetes, thyroid disease and connective tissue diseases. The inflamed lobules are reminiscent of the lympho-epithelial lesions seen in other auto-immune diseases e.g. Hashimoto's thyroiditis and Sjörgen's syndrome. ${ }^{6}$

Patients are diabetic, predominantly type 1 , on exogenous insulin. Many have complications of diabetes including retinopathy and microvascular disease. ${ }^{3}$ Other co-existing endocrine diseases may be present, especially thyroid disease. ${ }^{8}$

Most commonly, the patient presents with palpable change/s in the breast. The lumps may be multiple (67\% of patients) ${ }^{4}$ and bilateral (63\% of patients). ${ }^{3,4}$ The masses tend to be very firm, irregular, discrete and plaque-like ${ }^{3}$ but remain mobile. They may undergo rapid growth. ${ }^{4}$ The size of the mass ranges from $5-60 \mathrm{~mm}$. The clinical findings raise suspicion for breast carcinoma and patients are therefore referred for imaging.

Mammography generally does not demonstrate a distinct mass, spiculation or microcalcification. ${ }^{7}$ Most mammograms are reported as 'dense breast parenchyma', 'dense glandular tissue,' 'asymmetric densities' and 'parenchymal deformity."

US shows marked posterior acoustic shadowing from an irregular, hypo-echoic density. ${ }^{3}$ A small study demonstrated absence of Doppler signal on colour flow US9 (malignancies tend to show increased vascularity). US cannot distinguish this entity from malignancy. US should be used to guide core biopsy and to follow up these patients.

Scintimammography using Tc-99 sestamibi has been shown to be of use in palpable breast lesions. A few cases of diabetic mastopathy have demonstrated no radiotracer uptake with scintimammography; ${ }^{4}$ this requires further evaluation.

MRI is useful in assessing dense breast tissue and may be of use in evaluating suspected breast cancer in patients with diabetic mastopathy. ${ }^{2}$ Dynamic contrast MRI and MR spectroscopy may help support a diagnosis of diabetic mastopathy. There are few studies, however, in this regard; ${ }^{3}$ further studies are required to determine the role of MRI in this setting.

US-guided core biopsy is the preferred method to diagnose the condition. Fine needle aspiration yields insufficient cellular material in $50 \%$ of cases with diabetic mastopathy. ${ }^{3,10}$ The histological features are those of keloidal fibrosis and variable periductal, perilobular or perivascular lymphocytic infiltrate that consists predominantly of B-cells. Epitheliod fibroblasts have also been described. ${ }^{1,3,7}$ 


\section{CASE REPORT}

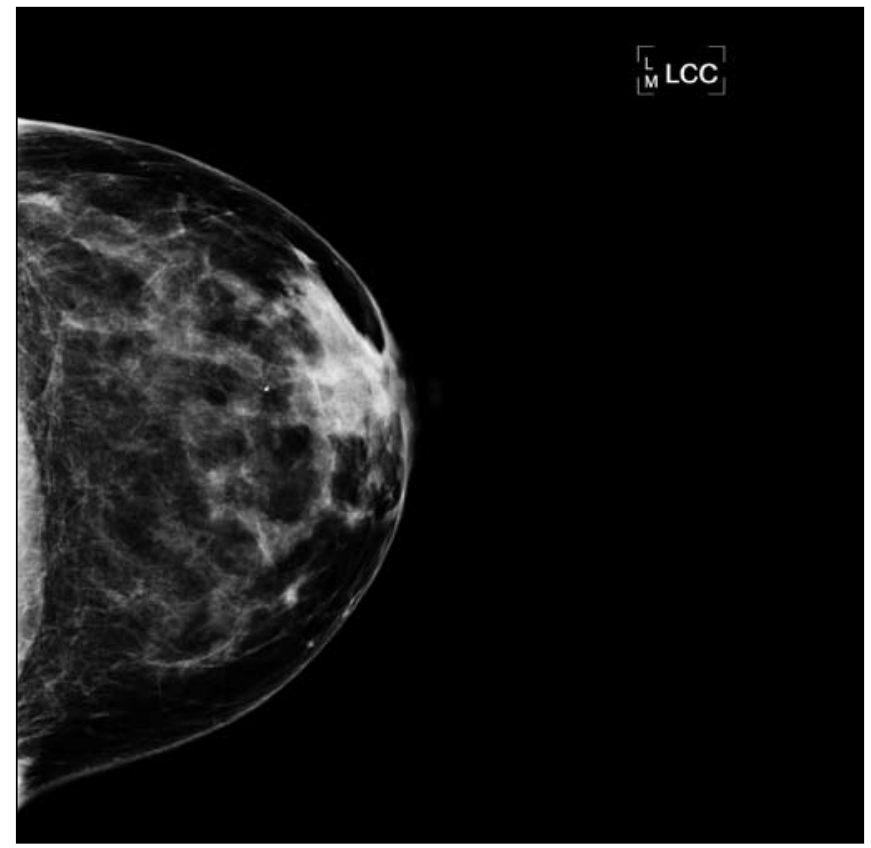

Fig. 1. Left craniocaudal mammogram.

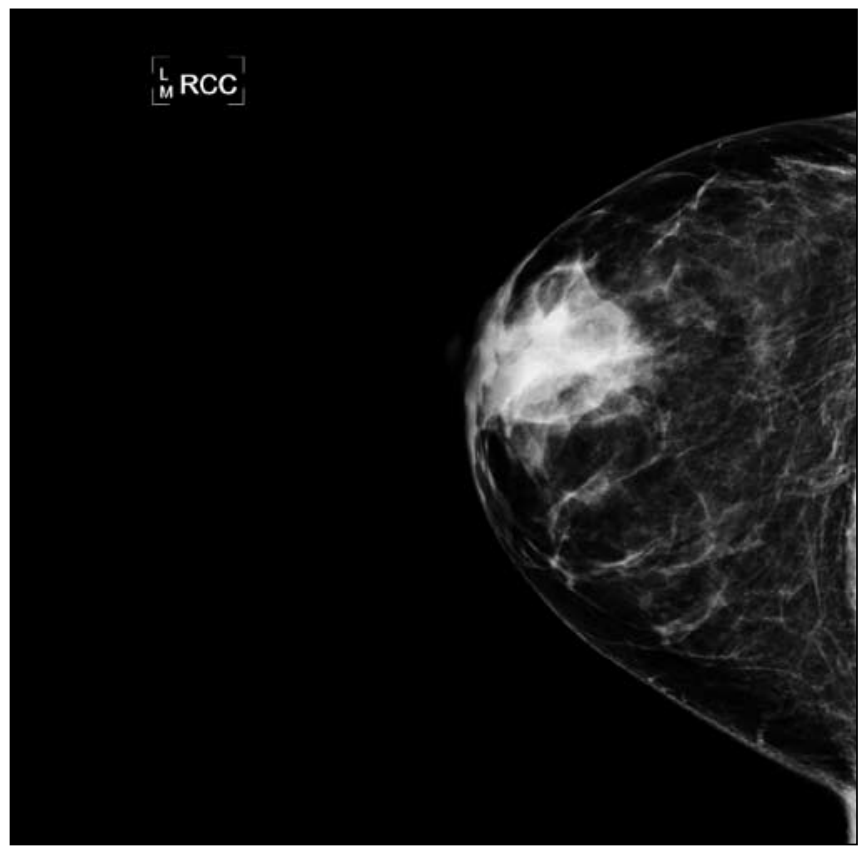

Fig. 2. Right craniocaudal mammogram.

The natural history of this disease is that $60 \%$ of the masses tend to be bilateral, recurrent or both - this reflects what appears to be a multicentric field effect of diabetes on mammary tissue. Surgery appears to exacerbate the condition. ${ }^{7,8}$ With the marked B-cell infiltrates and the presence of lympho-epithelial lesions, there has been concern that this disease carries a risk for lymphoma. However, all the studies concur that these patients are not at increased risk for lymphoma. ${ }^{1}$

Once this benign condition is diagnosed, the patient should perform routine breast self-examination and have regular clinical breast

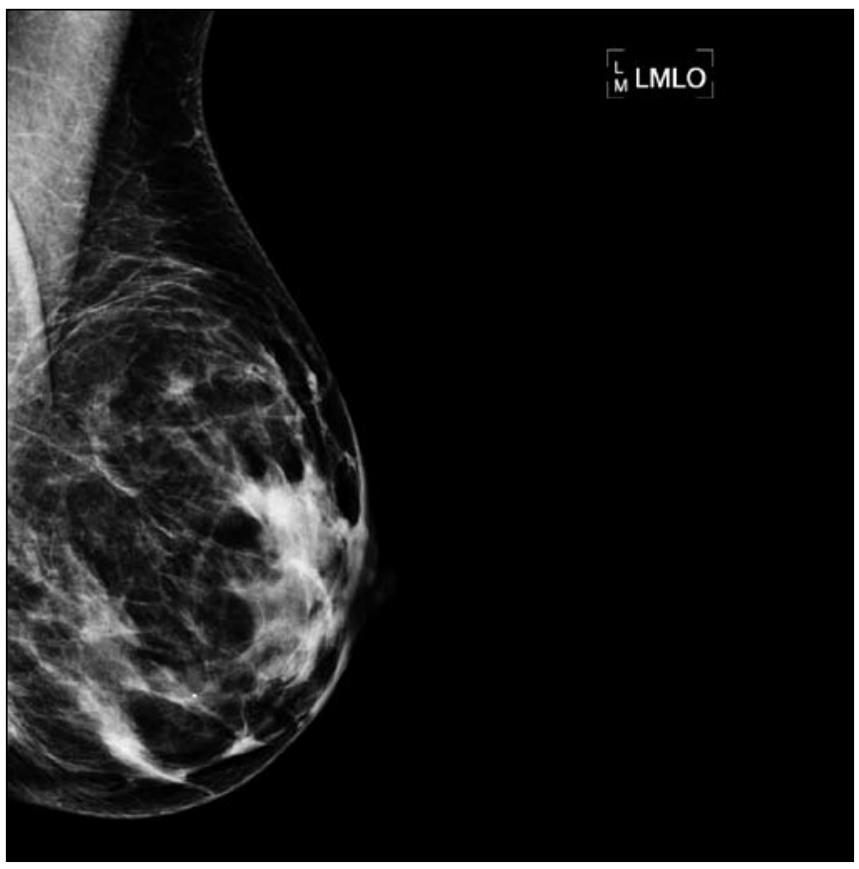

Fig. 3. Left mediolateral oblique view mammogram.

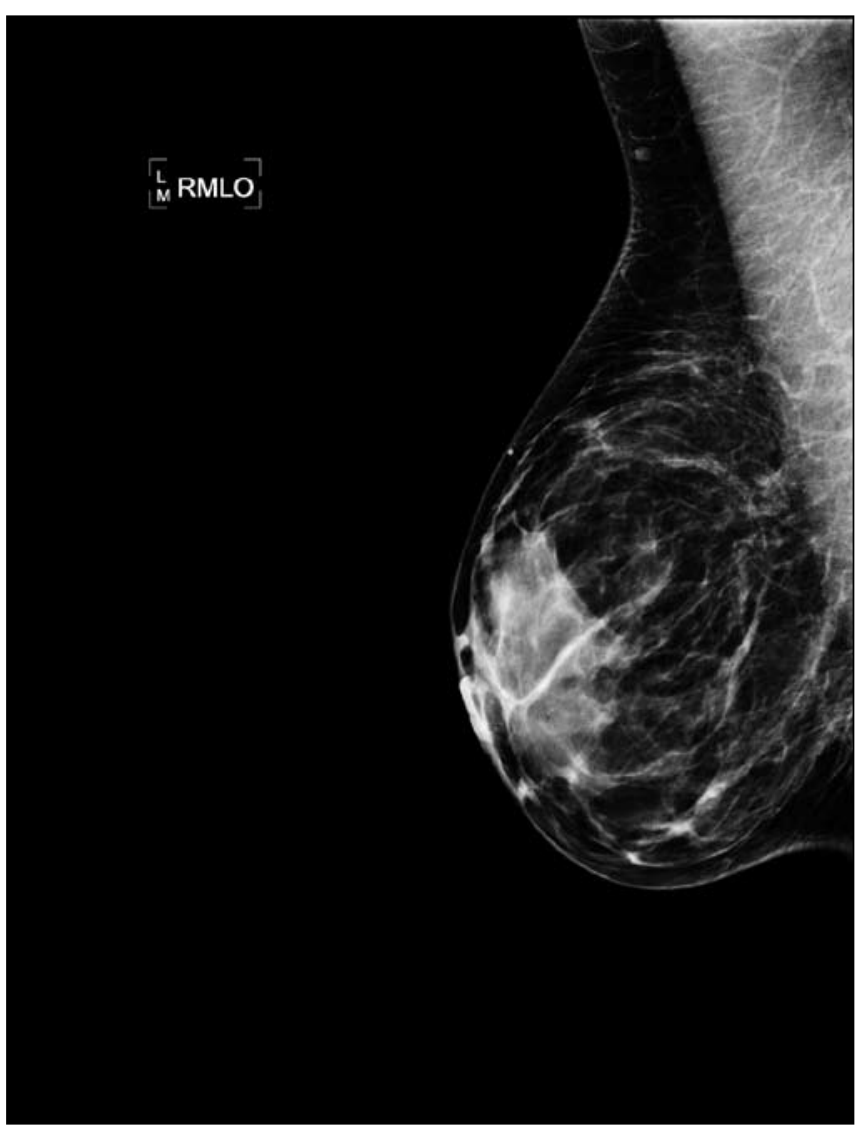

Fig. 4. Right mediolateral oblique view mammogram.

examinations. US and even MRI are recommended for follow-up. The minimum follow-up period is annual. ${ }^{7}$ If any changes are detected, core biopsy should be performed. ${ }^{3}$ 


\section{CASE REPORT}

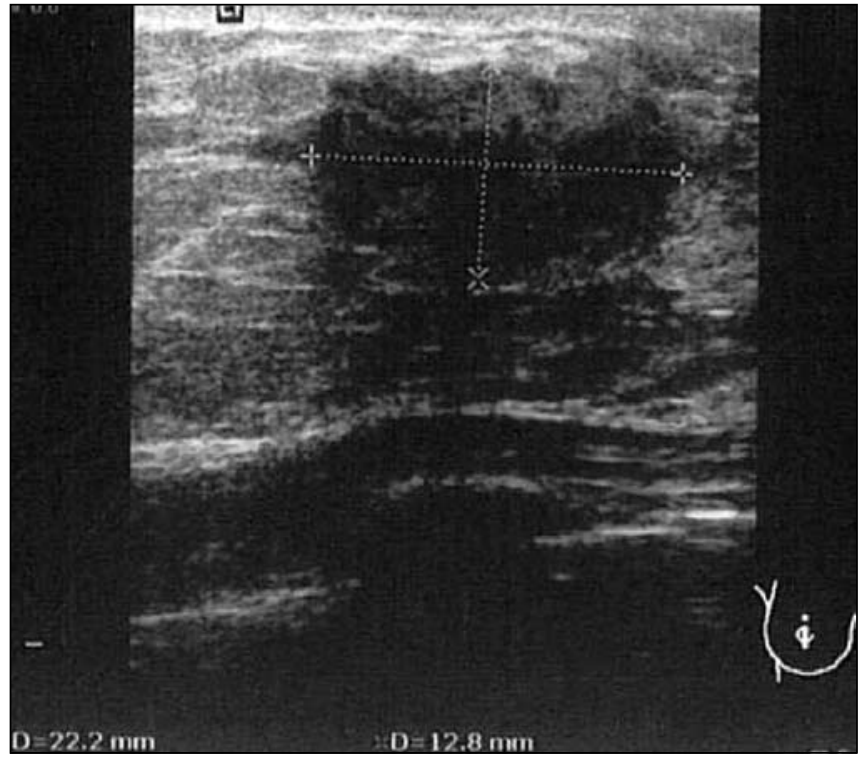

Fig. 5. Ultrasound image of the right breast.

\section{Conclusion}

Diabetic mastopathy is an uncommon but important entity. Core biopsy under US guidance is essential. Surgery should be avoided where possible, as it may unnecessarily exacerbate this benign condition.

1. Valdez R, Thorson J, William GF, et al. Lymphocytic mastitis and diabetic mastopathy: A molecular, immunophenotypic, and clinicopathological evaluation of 11 cases. Mod Pathol 2003; 16(3): 223-228.

2. Gabriel H, Feng C, Mendelson EB, Benjamin S. Breast MRI for cancer detection in a patient with diabetic mastopathy. AJR 2004; 182: 1081-1083.

3. Thorncroft K, Forsyth L, Desmond S, Audisio R.The diagnosis and management of diabetic mastopathy. Breast J 2007; 13(6): 607-613

4. Shaffrey J, Frederic BA, Gatewood O, Brem R. Diabetic fibrous mastopathy: case reports and radiologicpathologic correlation. Breast J 2008; 6(6): 414-417.

5. Tuncbilek N, Muammer Karakas H, Okten O. Diabetic fibrous mastopathy: dynamic contrast-enhanced magnetic resonance imaging findings. Breast J 2004; 10(4): 359-362.

6. Ely K, Tse G, Simpson J, Clarfeld R, Page DL. Diabetic mastopathy, a clinicopathological review. Am J Clin Pathol 2000; 113: 541-545.

7. Camuto P, Zetrenne E, Ponn T. Diabetic mastopathy. A report of 5 cases and a review of the literature. Arch Surg 2000; 135: 1190-1193.

8. Mak CW, Chou CK, Chen SY, Lee PS, Chang JM. Diabetic mastopathy. BJR 2003; 76: 192-194

9. Wong KT, Tse GMK, Yang WT. Ultrasound and MR imaging of diabetic mastopathy. Clin Radiol 2002; 57(8): 730-735.

10. Andrews-Tang D, Diamond AB, Rogers L, Butler D. Diabetic mastopathy: adjunctive use of ultrasound and utility of core biopsy in. Breast J 2001; 6(3): 183-188. 